\title{
Endovesical instillation of platelet rich fibrin for treatment of interstitial cystitis: case report of two patients
}

\author{
Falavolti Cristina1, Maria Cristina Tirindelli², Antonella Nicotera ${ }^{3}$, Tommasangelo Petitti ${ }^{4}$, Giuseppe Avvisati ${ }^{2}$, \\ Maurizio Buscarini ${ }^{5}$
}

\author{
${ }^{1}$ Department of Urology, Villa Betania Hospital of Rome, Via Pio IV 42, 00165 Rome, Italy. \\ ${ }^{2}$ Department of Hematology, Campus Biomedico University of Rome, Via Alvaro del Portillo 200, 00128 Rome, Italy. \\ ${ }^{3}$ Department of General Surgery, University of Turin, Via Giuseppe Verdi 8, 10124 Turin, Italy. \\ ${ }^{4}$ Hygiene and Public Health, Campus Biomedico University of Rome, Via Alvaro del Portillo 200, 00128 Rome, Italy. \\ ${ }^{5}$ Department of Urology, Campus Biomedico University of Rome, Via Alvaro del Portillo 200, 00128 Rome, Italy.
}

Correspondence to: Dr. Falavolti Cristina, Department of Urology, Villa Betania Hospital of Rome, Via Pio IV 42, 00165 Rome, Italy.

E-mail: c.falavolti@hotmail.com

How to cite this article: Cristina F, Tirindelli MC, Nicotera A, Petitti T, Avvisati G, Buscarini M. Endovesical instillation of platelet rich fibrin for treatment of interstitial cystitis: case report of two patients. Mini-invasive Surg 2017;1:186-90.

\begin{tabular}{l} 
Article history: \\
Received: 12 Jun 2017 \\
First Decision: 4 Aug 2017 \\
Revised: 21 Aug 2017 \\
Accepted: 28 Sep 2017 \\
Published: 28 Dec 2017 \\
\hline Key words: \\
Bladder pain syndrome, \\
cystoscopy, \\
interstitial cystitis, \\
platelet rich fibrin
\end{tabular}

\begin{abstract}
Interstitial cystitis, also called painful bladder syndrome, is a chronic condition causing bladder pain and sometimes pelvic pain. The exact cause of interstitial cystitis is not known. Often, signs and symptoms are hard to elucidate and no single treatment works for everyone. We report two cases of patients affected by interstitial cystitis treated with endovesical instillation of platelets rich fibrin (PRF). PRF is an autologous component that promotes angiogenesis, tissue growth and repair. This report presents the safety and the efficacy of PRF instillations in controlling clinical symptoms and restoring quality of life.
\end{abstract}

\section{INTRODUCTION}

Benign diseases of bladder such as interstitial cystitis (IC) and complex hemorrhagic radiation-induced cystitis are highly disabling. IC is not a specific disease but represents a complex set of symptoms also called "bladder pain syndrome". This differs from common bacterial cystitis as it is a non-infective bladder inflammation. IC is caused by cellular alteration of bladder wall. The etiology causing the mucosal damage is still unclear. The urothelium sustains multiple injuries in contact with the acidic urine and this leads to an increase in local nervous sensibility for pain regulation. Patients are more sensitive to voiding stimuli and suffer chronic pelvic pain, urgency and predisposition to urinary infections. These symptoms dramatically impact quality of life. Females are more affected than males. In $90 \%$ of cases, the patients are License (https://creativecommons.org/licenses/by/4.0/), which permits unrestricted use, distribution, and reproduction in any medium, as long as the original author is credited and the new creations are licensed under the identical terms.

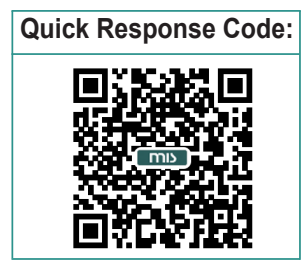


Table 1: Preoperative SF-36 questionnaire score

\begin{tabular}{lcc}
\hline Patient & $\begin{array}{c}\text { Preoperative SF-36 } \\
\text { score physical health } \\
\text { (baseline 50) }\end{array}$ & $\begin{array}{c}\text { Preoperative SF-36 } \\
\text { score mental health } \\
\text { (baseline 50) }\end{array}$ \\
\hline 1 & 35 & 25 \\
2 & 22 & 25 \\
\hline
\end{tabular}

SF-36: Short Form-36

20-40 years old. Prevalence is increasing. In 1999, the Nurses' Health Study has published a paper on over one million women affected by IC in the United States $^{[1]}$. Pathogenesis is still unclear and could be multifactorial including: urothelium dysfunction, mast cell activation and neurogenic inflammation ${ }^{[2]}$. Mast cells accumulate into the sub-urothelium, proliferate forming pericapillaries cluster and release numerous inflammatory molecules (histamine; cytokines; prostaglandins; platelet-activating factor and proteolytic enzymes (tryptase and kinase). The process may result in ulcerative cystitis ${ }^{[3]}$. The "upregulation" of bladder sensitive afferents is the cause of symptoms such as urgency, frequency and/or pain.

There is no standard treatment. The 2011 AUA Treatment Guidelines include a treatment protocol ranging from conservative treatments to more invasive interventions ${ }^{[4]}$. The aim of that study was to test the efficacy of platelets rich fibrin (PRF) in patients affected by IC in controlling the clinical symptoms and restoring the correct functioning of urothelium coating. $\mathrm{PRF}^{[5-9]}$ is a blood component for local use. It can be obtained from fresh frozen plasma or bought in synthetic form. It has hemostatic properties replicating the final phase of the coagulation cascade leading to the fibrin coat. PRF is composed of fibrin glue and threefold the number of platelets than normal human blood $^{[10]}$. PRF is stable and biocompatible. It is safe and functional. It promotes angiogenesis, tissue growth and repair through multiple growth factors such as transforming growth factor-beta, vascular endothelial growth factor and platelet-derived growth factor. PRF applications do not induce inflammatory processes, adverse reactions and tissue fibrosis. PRF is nowadays widely applied in different clinical scenarios, such as orthopedics, ophthalmology and healing therapies, as a growth factor pool for improving tissue regeneration.

\section{CASE REPORT}

The ethics committee of Campus Bio-Medico University of Rome approved the study (REC number: 27/14 PAR) on March 2014. From March 2014 to September 2014, we enrolled two women affected by IC who presented clinical symptoms such as pain, overactive bladder, dysuria, recurrent urinary infections previously treated by conventional drugs without any responses. The mean age was 60 years (range 5763 years). The exclusion criteria were: patients with Performance Status Karnofsky index $\leq 50 \%$; patients who needed major surgery; patients affected by cancer disease; patients presenting platelets counts $\leq 100,000$ or affected by coagulopathy; pregnancy. Both patients were negative for coagulation pathway alteration or urinary infection.

Patients underwent flexible diagnostic cystoscopy and biopsy 1 to 4 weeks before the application of PRF to exclude bladder cancer. One patient had a histopatological diagnosis of IC due to a previous transurethral resection of the bladder for suspicious carcinoma in situ of the bladder. Each patient filled in the urinary symptoms questionnaire for $I C^{[11-15]}$ and the SF (Short Form)-36 questionnaire ${ }^{[16-18]}$ before the endoscopic procedure and application of PRF. The urinary symptoms questionnaire was composed of 73 questions covering the following domains: urinary symptoms, pain symptoms, sexual function, menstrual variability, and general health status. The SF-36 questionnaire was used to test the quality of life, using a baseline of 50 represented the general health of the unaffected Italian population. The results of SF36 questionnaire are shown in Table 1. Summary score of IC symptom index was higher than 6 for both patients. This score indicates that patients affected by IC present significant voiding and pain symptoms.

After the written informed consent was obtained, we took $120 \mathrm{~mL}$ of autologous blood sample about $1 \mathrm{~h}$ before the procedure from a peripheral vein $(30 \mathrm{~min}$ is the time for preparing about $6 \mathrm{~mL}$ of PRF). PRF was obtained from autologous patient's blood through the Vivostat system (Vivolution, Birkeroed, Denmark ${ }^{[19]}$ according to the standards for autologous donation (Ministerial Decrees of March 2005). Vivostat system is composed of 3 parts: an automated processor unit, an automated applicator unit and a sterile unit for dispensing which includes a set of preparation and an endoscopic applicator. PRF was controlled for hepatitis B virus, hepatitis C virus, and human immunodeficiency virus. We obtained about $6 \mathrm{~mL}$ of PRF from each patient through the Vivostat system. Thereafter, we performed an operative rigid cystoscopy with Carbon Dioxide Insufflation. One patient was treated under sedation, the other one received subarachnoid anesthesia due to serious asthma condition. We instilled $6 \mathrm{~mL}$ of PRF in a spray fashion all over the bladder walls [Figures 1 and 2] through the endoscopic applicator. Then we placed the vesical catheter and monitored the urine output. 


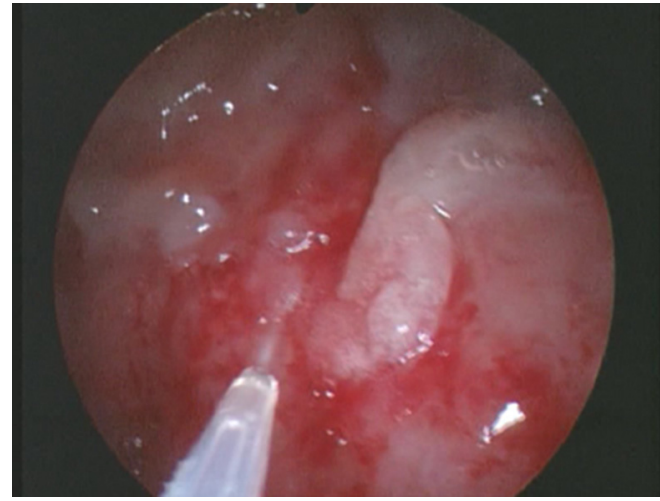

Figure 1: Instillation of platelets rich fibrin during operative cystoscopy

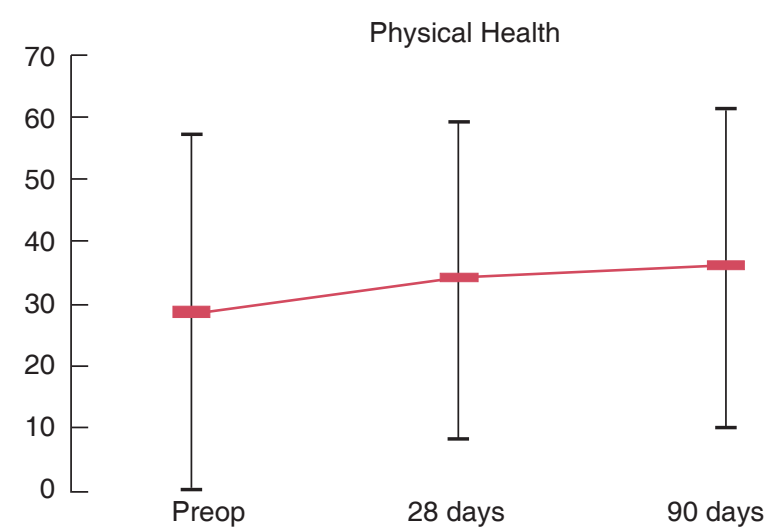

Figure 3: Physical health improvement after platelets rich fibrin instillation (Short Form-36 questionnaire score)

Both patients maintained the vesical catheter postoperatively and presented good urinary output. We discharged patients after removal of the catheter and spontaneous urination.

The follow-up was performed at 28 days, and 3 months through clinical visits and patients were asked to fill in the SF-36 questionnaire again. During the last follow-up at 3 months, patients filled in also the Patient Global Impression of Improvement (PGI-I) questionnaire $^{[20]}$. PGI-I is a 1-item questionnaire designed to assess the patient's impression of changes in urinary symptoms on a 7-point scored scale. Global clinical response was evaluated through clinical examinations and postoperative questionnaires that patients filled in during the follow-up. Patients reported significant improvement of symptoms (pain and dysuria) and quality of life especially regarding the mental health at the SF-36 questionnaire [Figures 3 and 4]. Both patients answered the PGI-I questionnaire to feel "much better" after PRF instillation. Neither patient treated with PRF experienced any short- or long-term side effects.

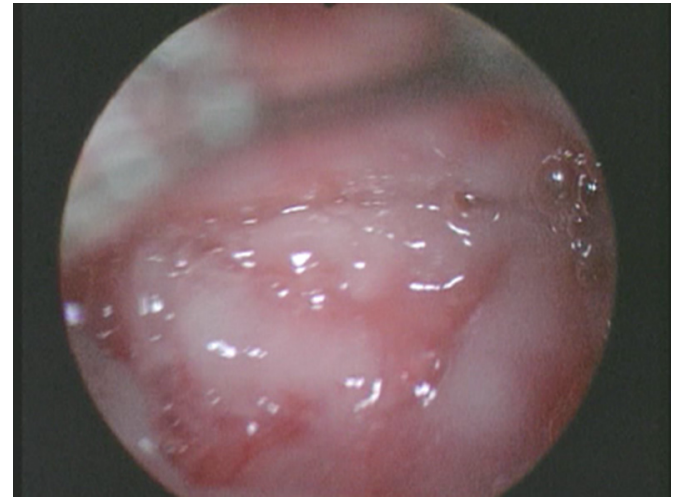

Figure 2: Appearance of bladder walls at the end of the procedure

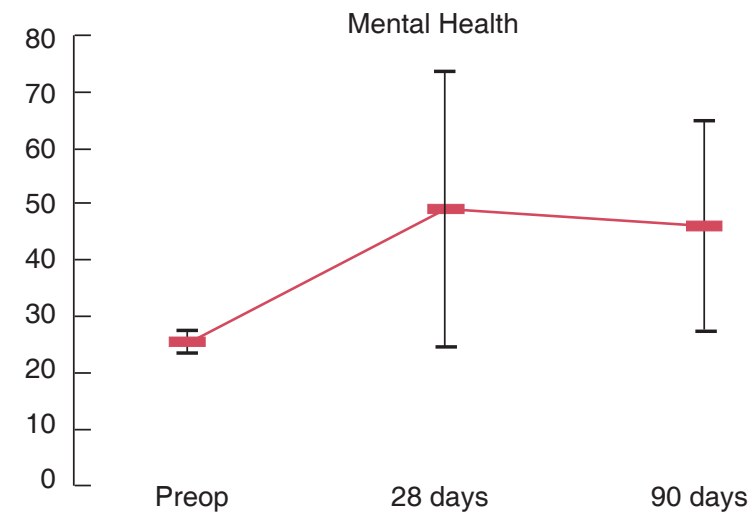

Figure 4: Mental health improvement after platelets rich fibrin instillation (Short Form-36 questionnaire score)

\section{DISCUSSION}

During the 2003 Kyoto workshop, the IC was defined to be a syndrome characterized by 3 main symptoms: frequency; urgency; pain ${ }^{[21]}$. The discomfort ${ }^{[22]}$ significantly alters the quality of life of patients. The etiology and pathogenesis of this condition are still unknown and it remains diagnosed by exclusion. According to the European Society for the study of IC/BPS (ESSIC) recommendations ${ }^{[23]}$, the first line of diagnosis is patient selection based on symptoms and exclusion of other diseases with similar presentation. During the cystoscopy, little pink ulcerations (ulceration of Hunner ${ }^{[24]}$ ) described in 1914 and "glomerulation" described by Keene in 1920 as sub-mucosal capillary bleeding can be found. The histologic findings are also neither specific for diagnosis nor correlated with symptoms. The histologic sample of bladder biopsy can show the coating involution, the urothelium thinning, the inflammatory sub-urothelium infiltration and the mast cells presence ${ }^{[25]}$. While a patient's history and examination are important, bladder biopsy is not essential. The most common indication for 
bladder biopsy is a search for urothelial carcinoma/ carcinoma in situ of the bladder, which may be confused with $\mathrm{IC}^{[26]}$.

We enrolled patients according to their typical and highly disabling symptoms. Once we excluded the presence of bladder cancer, the aim of this study was to test the safety and the efficacy of endovesical instillation of PRF to stimulate tissue regeneration and control the clinical symptoms. Because standardized treatment for IC does not exist, patients usually try many kinds of procedures such as many drugs (pentosan polysulphate, antidepressants); endovesical instillation of hyaluronic acid or Bacillus Calmette-Guerin; sacral neuromodulation or surgery (laser ablation of ulceration, cystectomy, bladder augmentation). Instillation therapy has a direct action on the urothelium and sub-urotheliium to restore the normal bladder lining. Endovesical fibrin glue has been succesfully used also for refractory hemorrhagic cystitis occurred after unrelated marrow, cord blood, and haploidentical hematopoietic stem cell transplantation ${ }^{[27]}$. Campus Bio-Medico University (Urology and Hematology Departments) has already conducted, as coordinating center of Rome Transplanta Network in 2009, a study on 35 patients affected by severe hemorrhagic cystitis after allogeneic hematopoietic stem cell transplantation. All 35 patients showed clinical response and pain resolution. In this new study, we treated patients affected by IC and we demonstrated that the typical urothelium coating involution can be successfully helped by PRF that promotes angiogenesis, tissue growth and repair through multiple growth factors as showed for transplanted patients.

A significant number of patients with ulcerative IC show ulceration, severe inflammation, and granulation tissue ${ }^{[28]}$. The inflammatory infiltrates are usually superficial, and restricted to the lamina propria ${ }^{[29]}$. The lamina propria is edematous, with stromal hemorrhage and congested venules. In these patients, the rupture of the bladder mucosa subsequently resulted in reparative granulation tissue ${ }^{[30]}$. Hemorrhage is present in $90 \%$ of nonulcerative IC. While generally localized, the hemorrhage may extend into the urothelium ${ }^{[25]}$ and causes the mucosal rupture. Usually inflammation is mild, but edema and vascular congestion are frequently seen. In both cases (ulcerative and non-ulcerative IC) the urothelium is particularly fragile. Baseline symptom assessment and regular symptom level reassessment are essential to document efficacy of single and combined treatments ${ }^{[31]}$. We used different types of questionnaires to compare the entity of patients' discomfort before and after the endoscopic application of PRF. Questionnaires score showed a significant improvement of patients' symptomatology during the follow-up. Our patients presented improvement in quality of life and symptoms especially regarding the mental health. Both the mental and the physical health increased significantly and remained stable after 28 days and 3 months. PGI-I and SF-36 questionnaires can provide an overall appraisal of a patients' condition and they are practical for clinical use by their simplicity in administration and interpretability. The interstitial cystitis symptom index has been designed to capture the most important voiding and pain symptoms and to assess how problematic patients find them. Almost no IC patients score less than 6 while almost no controls score as high as 6 . In our study, the symptom index was higher than 6 for both patients and this value is comparable to the score of O'Leary et al. ${ }^{[11]}$. No further clinical improvements were seen after the first weeks after PRF instillation. This may justify revaluation during the follow-up for new endovesical treatments even though they presented partial or no response. PRF is an autologous blood component and it is safe and biocompatible. Patients treated with PRF did not present any immediately or long term side effects. The small series represents the major limitation of the study. Further cases are needed to achieve stronger results.

In conclusion, PRF is an autologous component that promotes angiogenesis, tissue growth and repair through multiple growth factors. It is safe and biocompatible; it is widely applied in different clinical scenarios to promote the tissue regeneration. Our preliminary data show that patients affected by IC who underwent endovesical application of PRF over the damaged urothelium, present significant improvement in clinical symptoms and quality of life. Further studies are needed to obtain more data.

\section{DECLARATIONS}

\section{Authors' contributions}

Study conception and design: F. Cristina

PRF production: M.C. Tirindelli

Data collection: A. Nicotera

Analysis and interpretation of data: T. Petitti

Drafting of manuscript: M. Buscarini

Critical revision: G. Avvisati

\section{Financial support and sponsorship} None.

\section{Conflicts of interest}

There are no conflicts of interest. 


\section{Patient consent \\ Obtained from both patients.}

\section{Ethics approval}

The ethics committee of Campus Bio-Medico University of Rome approved the study (REC number: 27/14 PAR) on March 2014.

\section{REFERENCES}

1. Curhan GC, Speizer FE, Hunter DJ, Curchan SG, Stampfer MJ. Epidemiology of interstitial cystitis: a population based study. $J$ Urol 1999;161:549-52.

2. Teichman JM, Moldwin R. The role of the bladder surface in interstitial cystitis/painful bladder syndrome. Can J Urol 2007;14:3599-607.

3. Fall M, Johansson SL, Aldenborg F. Chronic interstitial cystitis: a heterogeneous syndrome. J Urol 1987;137:35-8.

4. American Urological Association (AUA) treatment guidelines 2011. Available from: http://www.auanet.org/guidelines [Last accessed on 4 Dec 2017]

5. Mankad PS, Codispoti M. The role of fibrin sealants in hemostasis. Am J Surg 2001;182:S21-8.

6. Jackson MR. Fibrin sealants in surgical practice: an overview. Am J Surg 2001;182:S1-7.

7. Schwartz M, Madariaga J, Hirose R, Shaver TR, Sher L, Chari R, Colonna JO 2nd, Heaton N, Mirza D, Adams R, Rees M, Lloyd D. Comparison of a new fibrin sealant with standard topical hemostatic agents. Arch Surg 2004;139:1148-54.

8. Fattahi T, Mohan M, Caldwell GT. Clinical applications of fibrin sealants. J Oral Maxillofac Surg 2004;62:218-24.

9. Hong YM, Loughlin KR. The use of hemostatic agents and sealants in urology. J Urol 2006; 176:2367-74.

10. Serrano Frago P, Allepuz Losa C, Gil Martínez P, Allué López M, Mallén Mateo E, Sancho Serrano C, Rioja Sanz LA. Treatment of hemorrhagic cystitis secondary to cyclophosphamide. Literature review with regard to a case. Actas Urol Esp 2005;29:230-3. (in Spanish)

11. O'Leary MP, Barry MJ, Fowler FJ Jr. Hard measures of subjective outcomes: validating symptom indexes in urology. $J$ Urol 1992;148:1546-8, discussion 1564.

12. Keller ML, McCarthy DO, Neider RS. Measurement of symptoms of interstitial cystitis. A pilot study. Urol Clin North Am 1994;21:67-71.

13. Kleinbaum DG, Kupper LL, Morgenstem H. Epidemiologic Research: Principles and Quantitative Methods. New York: Lifetime Learning Publications; 1982. p. 152.

14. Deyo RH, Diehr P, Patrick D. Reproducibility and responsiveness of health status measures. Statistics and strategies for evaluation. Controlled Clin Trials 1991;12:S142-58.
15. Cronbach LJ. Coefficient alpha and the internal structure of tests. Psychometrika 1951;16:297A.

16. Wasson JH, Reda DJ, Bruskewitz RC, Elinson J, Keller AM, Henderson WG; Veterans Affairs Cooperative Study Group on Transurethral Resection of the Prostate. A comparison of transurethral surgery with watchful waiting for moderate symptoms of benign prostatic hyperplasia. N Engl J Med 1995;332:75-9.

17. Ware JE Jr, Sherbourne CD. The MOS 36-item short-form health survey (SF-36). I. Conceptual framework and item selection. Med Care 1992;30:473-83

18. Apolone G, Mosconi P, Ware JE. Questionario sullo stato di salute SF-36. Manuale d'uso e guida all'interpretazione dei risultati. Milano: Guerini e associati; 1997.

19. Dodd RA, Cornwell R, Holm NE, Garbarsch A, Hollingsbee DA. The Vivostat application system: a comparison with conventional fibrin sealant application systems. Technol Health Care 2002;10:401-11.

20. Srikrishna S, Robinson D, Cardozo L. Validation of the Patient Global Impression of Improvement (PGI-I) for urogenital prolapse. Int Urogynecol J 2010;21:523-8.

21. Diokno AC, Homma Y, Sekiguchi Y, Suzuki Y. Interstitial cystitis, gynecologic pelvic pain, prostatitis, and their epidemiology. Int J Urol 2003;10: S3-6

22. Offiah I, McMahon SB, O'Reilly BA. Interstitial cystitis/bladder pain syndrome: diagnosis and management. Int Urogynecol J 2013;24:124356.

23. van de Merwe JP, Nordling J, Bouchelouche P, Bouchelouche $\mathrm{K}$ Cervigni M, Daha LK, Elneil S, Fall M, Hohlbrugger G, Irwin P, Mortensen S, van Ophoven A, Osborne JL, Peeker R, Richter B, Riedl C, Sairanen J, Tinzl M, Wyndaele JJ. Diagnostic criteria, classification, and nomenclature for painful bladder syndrome/interstitial cystitis: an ESSIC proposal. Eur Urol 2008;53:60-7.

24. Hunner GL. A rare type of bladder ulcer in women: report of cases. Trans South Surg Gynecol Assoc 1914;27:247-92.

25. Johansson SL, Fall M. Clinical features and spectrum of light microscopic changes in interstitial cystitis. J Urol 1990;143:1118-24.

26. Lamm DL, Gittes RF. Inflammatory carcinoma of the bladder and interstitial cystitis. J Urol 1977;117:49-51.

27. Tirindelli MC, Flammia G, Sergi F, Cerretti R, Cudillo L, Picardi A, Postorino M, Annibali O, Greco R, Avvisati G, Arcese W. Fibrin glue for refractory hemorrhagic cystitis after unrelated marrow, cord blood, and haploidentical hematopoietic stem cell transplantation. Transfusion 2009;49:170-5.

28. Cheng L, Lopez-Beltran A, Bostwick DG. Bladder pathology. In: Interstitial cystitis. Hoboken (NJ): Wiley-Blackwell; 2012. p. 22-5.

29. Lynes WL, Flynn SD, Shortliffe LD, Stamey TA. The histology of interstitial cystitis. Am J Surg Pathol 1990;14:969-76.

30. Kim HJ. Update on the pathology and diagnosis of interstitial cystitis/ bladder pain syndrome: a review. Int Neurourol J 2016;20:13-7.

31. American Urological Association (AUA) treatment guidelines 2016. Available from: http://www.auanet.org/guidelines [Last accessed on 4 Dec 2017] 